\title{
Hubble's Next Generation Spectral Library
}

\author{
Sara R. Heap ${ }^{1}$ \& Don Lindler ${ }^{1,2}$ \\ ${ }^{1}$ NASA's Goddard Space Flight Center, Greenbelt MD 20771, USA \\ ${ }^{2}$ Sigma Space Corp., 9801 Greenbelt Rd., Lanham MD 20706, USA
}

\begin{abstract}
We give a progress report on our work to reduce and calibrate spectra obtained for Hubble's Next Generation Spectral Library (NGSL). We will shortly be working with Bruzual and Charlot to incorporate these spectra into their stellar population synthesis code.
\end{abstract}

Keywords. stars: fundamental parameters, ultraviolet: stars, space vehicle: instruments, techniques: spectroscopic, atlases

\section{Introduction}

Hubble's Next Generation Spectral Library (NGSL; PI=Gregg) comprises intermediate-resolution $(R \sim 1000)$ STIS spectra of 378 stars having a wide range in metallicity and age. Unique features of the NGSL include its broad wavelength coverage $(1,800-$ $10,100 \AA$ ) and high-S/N, absolute spectrophotometry. When incorporated in Bruzual \& Charlot's (2003) stellar population synthesis code, it should enable us to constrain simultaneously the star-formation history and light-weighted mean age, stellar metallicity, and mass of galaxies over a wide redshift interval $(z=0-2)$. This comes about because both mid-UV and optical spectral indices can be used, thereby increasing the redshift interval of their application, and because the same stars are used as spectral templates so that low- and high-redshift galaxies are treated consistently.

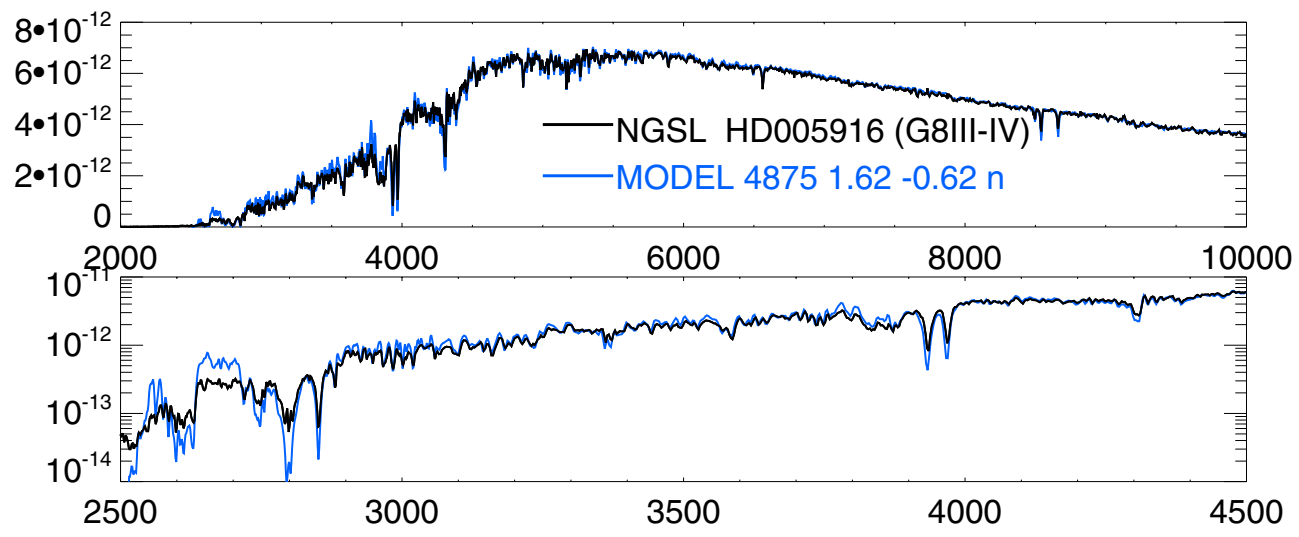

Figure 1. Comparison of Munari model to NGSL spectrum of HD 5916.

\section{NGSL Spectra and Spectral Indices}

Figure 1 shows the spectrum of a sample star in the NGSL. It compares the calibrated spectrum of HD 5916 to one of Munari et al.'s (2005) high-resolution model spectra (blurred and binned to the resolution and sample size of STIS). The model parameters ( $\mathrm{T}_{\mathrm{eff}}, \log g, \log Z$, and indications of $\alpha$ enhancement) were derived from $\chi^{2}$-fitting the 

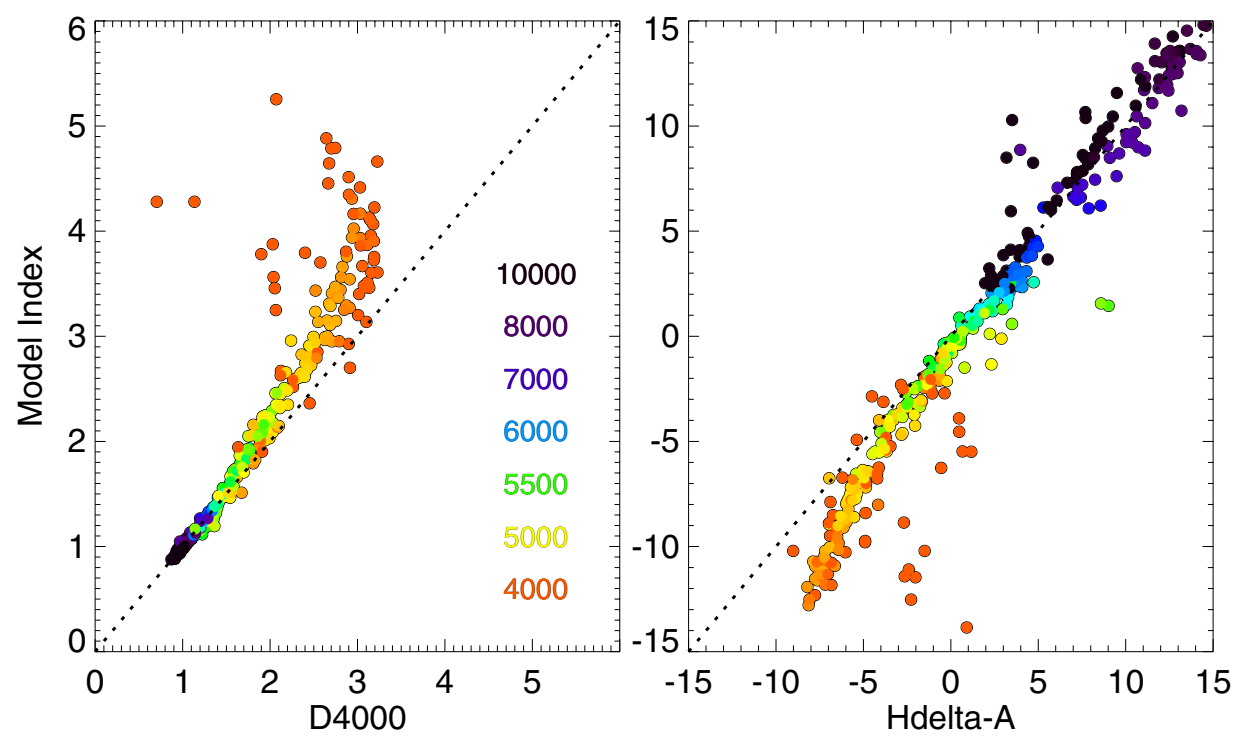

Figure 2. Comparison of observed and model indices, D4000 and $\mathrm{H} \delta_{A}$. The star symbols are color-coded by effective temperature.

observed spectrum over the spectral range, 0.3-1.0 microns. The full spectrum fitting produces excellent wide-baseline SED's, which are essential for breaking the dust-agemetallicity degeneracy.

The lower panel of Figure 1 gives detail on the UV-blue portion of the spectrum of HD 5916. It shows that the model fit is poor below $3000 \AA$, especially at the $2640-\AA$ break, and only fair at the $4000-\AA$ break. Poor model fits pose a vexing problem for population synthesis: observed spectra of stars covering the HR diagram at all metallicities are rare or non-existent, but model spectra are unreliable due to incomplete atomic data.

To address this problem, we are deriving semi-empirical spectral indices by comparing observed and model spectral indices and working out the corrections to the models that must be applied at various temperatures, gravities, and metallicities. We have already done so for the two indices used by Kauffmann et al. (2003), D4000 and $\mathrm{H} \delta_{A}$, with encouraging results as shown in Figure 2. This figure confirms that for cool stars $\left(\mathrm{T}_{\text {eff }} \leqslant\right.$ $5000 \mathrm{~K})$, the models tend to overestimate the strength of the CaII $\lambda 4000$ doublet, and they overestimate the depression of the apparent continuum in the vicinity of $\mathrm{H} \delta$ by metal-line absorption, thereby producing a spuriously strong "emission" line.

We plan to work with Bruzual and Charlot in incorporating the calibrated NGSL spectra into their population synthesis code.

\section{Acknowledgements}

This research was supported by Hubble program, AR10659.

\section{References}

Bruzual, A.G. \& Charlot, S. 2003, MNRAS, 344, 1000

Heap, S.R. \& Lanz, T.M. 2005, in Multiwavelength Mapping of Galaxy Formation and Evolution, Springer-Verlag: Berlin, p. 210

Kauffmann, G., Heckman, T., White, S., et al. 2003, MNRAS, 341, 54

Munari, U. et al. 2005, A\& A, 442, 1127 